\title{
Synthesis of Th17 cytokines in the culture of peripheral blood mononuclear cells stimulated with Borrelia burgdorferi sensu lato
}

\author{
Sambor Grygorczuk1, Renata Świerzbińska', Anna Moniuszko', Maciej Kondrusik', \\ Joanna Zajkowska', Piotr Czupryna', Justyna Dunaj', Sławomir Pancewicz' \\ ${ }^{1}$ Department of Infectious Diseases and Neuroinfections, Medical University, Białystok, Poland \\ Grygorczuk S, Świerzbińska R, Moniuszko A, Kondrusik M, Zajkowska J, Czupryna P, Dunaj J, Pancewicz S. Synthesis of Th17 cytokines in the \\ culture of peripheral blood mononuclear cells stimulated with Borrelia burgdorferi sensu lato. Ann Agric Environ Med. 2016; 23(2): 242-247. \\ doi: $10.5604 / 12321966.1203884$
}

\begin{abstract}
Introduction and objective. Th17 lymphocytes and their cytokines, interleukin 17A (IL-17A), IL-17F and IL-22, participate in the response to extracellular bacteria and in the autoimmunity and may be engaged in the pathogenesis of Lyme borreliosis. Concentrations were measured of IL-17A, IL-17F and IL-22 in the supernatant of the peripheral blood mononuclear cells (PBMC) culture stimulated with Borrelia burgdorferi sensu lato (B. burgdorferi).

Materials and method. The study group consisted of 13 patients with early disseminated and late Lyme borreliosis and a control group of 7 healthy persons. PBMC cultures were stimulated for 48 hours with $B$. burgdorferi spirochetes of three pathogenic species: $B$. burgdorferi sensu stricto, B. afzelii or B. garinii, in the multiplicity of infection 10:1. Concentrations of Th17 cytokines IL-17A, IL-17F and IL-22, as well as Th2/immunoregulatory cytokine IL-10 were measured with ELISA assays. Results. Expression of IL-17A, IL-17F and IL-22 increased under stimulation, simultaneously with the increased IL-10 expression. Concentration of IL-17F tended to be lower in early neuroborreliosis than in late Lyme borreliosis and than in controls. B. afzelii elicited higher expression of IL-17A than the other two species.

Conclusions. IL-17A, IL-17F and IL-22 are synthesized simultaneously by PBMC stimulated with B. burgdorferi. There is no antagonism between Th17 response and IL-10 expression. The role of Th17 cytokines seems to differ depending on the clinical stage of Lyme borreliosis and on the B. burgdorferi species.
\end{abstract}

Key words

Lyme borreliosis, Th17 lymphocytes, inflammation, cytokines, Borrelia burgdorferi genospecies

\section{INTRODUCTION}

Lyme borreliosis is an infectious disease caused by the tick-borne spirochete Borrelia burgdorferi sensu lato (B. burgdorferi), presenting with a variable clinical picture, affecting the skin, nervous and/or musculoskeletal system. It is usually self-limited or resolves after antibiotic therapy, but late neurologic and articular manifestations may be, for not fully explained reasons, antibiotic-refractory $[1$, $2,3,4]$. Some clinical forms, such as primary infection (erythema migrans, EM), early neuroborreliosis and atrophic dermatitis (acrodermatitis chronica atrophicans, ACA), are caused by an active infection which may be selflimiting (EM, early neuroborreliosis) or persist for years (ACA). Other manifestations, e.g. Lyme arthritis caused by Borrelia burgdorferi sensu stricto (B. burgdorferi ss), have an inflammatory/autoimmune component and may continue after eradication of the infection $[1,3,4]$. Thus, a clinically unfavourable outcome may depend both on the inability to clear the infection and on the secondary inflammatory complications. This picture is complicated by the variable pathogenetic and pro-inflammatory properties of three pathogenic B. burgdorferi sl species: B. burgdorferi ss, B. garinii and B. afzelii, with the most invasive infection and the strongest inflammatory response caused by $B$. burgdorferi ss [5].

Address for correspondence: Sambor Grygorczuk, Department of Infectious Diseases and Neuroinfections, Medical University, Białystok, Poland

E-mail: grygorczuk.sambor@neostrada.pl

Received: 09 December 2014; accepted: 13 January 2015
Th17 lymphocytes form a distinct, strongly proinflammatory subpopulation of Th lymphocytes, normally constituting of less than $1 \%$ of the peripheral blood mononuclear cell (PBMC) population $[6,7,8,9]$. There is an antagonism between Th17 and other types of Th responses, with Th1 (interferon $\gamma$ ) and Th2 (Il-4, Il-13) cytokines inhibiting the development of Th17 phenotype $[9,10,11]$. Th17 cells play a dominant role in neutrophilic inflammation and in response to extracellular pathogens, especially on the skin and mucous membranes $[7,9,12]$. Their influence is exerted through a set of specific cytokines, including IL17A, IL-17F and IL-22 [7]. IL-17A is responsible for most of the biologic effects of Th17 lymphocytes, acting through a multimer membrane receptor composed of IL-17RA and IL-17RC subunits [7]. Its homologue IL-17F binds the same receptor and has an overlapping and synergistic, although weaker, effect on target cells [12]. IL-22 is a IL-10 homologue secreted by Th17 cells, together with IL-17A and IL-17F, and by a specialized subpopulation of Th22 lymphocytes $[9,13]$. In a pro-inflammatory environment it acts synergistically with IL-17A and participates in the antibacterial defence [9].

There is growing evidence that Th17-type response participates in B. burgdorferi-induced inflammation [6, 11, $14,15]$. It is also involved in autoimmunity and may be crucial in the pathogenesis of different forms of autoimmune arthritis and in sclerosis multiplex $[10,12,16]$. This might be relevant for the pathogenesis of the clinical forms of Lyme borreliosis characterized by uncontrolled inflammation within the nervous system or joints, as in the antibioticrefractory Lyme arthitis. However, the role of Th17 cytokines 
in the particular manifestations of Lyme borreliosis and in the response to different $B$. burgdorferi species is not well known. Most studies have concentrated on IL-17A, while there are few or no data on other Th17 cytokines.

\section{OBJECTIVE}

The expression was studied of IL-17A, IL-17F and IL-22 in PBMC cultures from healthy persons and patients with different Lyme borreliosis manifestations, stimulated with spirochetes of three pathogenic B. burgdorferi species. The results were compared with the simultaneous synthesis of the Th2-related and immunoregulatory cytokine IL-10, which has been postulated to influence the Th17 response in that setting [13].

\section{MATERIALS AND METHOD}

13 patients with disseminated Lyme borreliosis were studied: 3 with Lyme arthritis (one man aged 57, two women, aged 35 and 41), 7 with neuroborreliosis and 3 with ACA (3 men aged 27, 68 and 75). The diagnosis was based on the epidemiologic data (tick bites or frequent exposition to ticks in the endemic areas), clinical presentation and confirmed serologically. Patients with Lyme arthritis had recurrent asymmetric artralgia and mild arthritis of the large peripheral joints. The patients with ACA had typical cutaneous foci; all of them were consulted by a dermatologist as a part of differential diagnosis, and a histopathology of a skin sample was performed, with the result supporting the diagnosis of ACA. All the patients with Lyme arthritis and ACA were diagnosed with late Lyme borreliosis, based on the reported duration of symptoms. Of patients with neuroborreliosis, 5 had symptoms of early disseminated Lyme borreliosis: subacute meningitis with predominantly lymphocytic pleocytosis in 3 (2 men aged 19 and 66, and a woman aged 63), facial and oculomotor paresis with menigneal signs in one man aged 70 . In the latter patient, csf was not available for general and serologic examination due to his lack of consent for the diagnostic lumbar puncture, but the clinical picture was consistent with early csf neuroborreliosis. In the remaining patient, a woman aged 55, there was also involvement of the VIIIth cranial nerve with no changes in csf general examination The remaining 2 neuroborreliosis patients had had symptoms for over 1 year and were diagnosed with late disease: one (a man aged 75) presented with polineuropathy and the other (a man aged 63) with hemiparesis and increased cerebrospinal fluid (csf) protein concentration, suggesting late central nervous system (cns) neuroborreliosis. The appropriate differential diagnostics was performed in all the patients to exclude infectious and inflammatory co-morbidity (e.g. testing for rheumatoid factor in patients with arthritis and diagnostics towards alternative causes of the lymphocytic meningitis).

Anti-B. burgdorferi antibodies were detected in serum obtained on admission with ELISA assay from Biomedica (Boston, USA), containing the recombinated antigens B. burgdorferi ss $\mathrm{p} 21$ (OspC), B. garinii $\mathrm{p} 41$ and B. afzelii $\mathrm{p} 41$ for the detection of the specific IgM antibodies and p21, B. garinii $\mathrm{p} 41$, B. afzelii $\mathrm{p} 41, \mathrm{p} 18$ and $\mathrm{p} 100$ for the detection of specific IgG. The results of the test are expressed in Biomedica Borrelia Units (BBU) per $\mathrm{ml}$, with $>11 \mathrm{BBU} / \mathrm{ml}$ considered positive, $>20 \mathrm{BBU} / \mathrm{ml}$ high positive and $>30 \mathrm{BBU} / \mathrm{ml}$ - very high positive. All the patients had positive IgG serology, in early Lyme borreliosis accompanied by positive IgM, and all the patients with late Lyme borreliosis were all IgG high/ very high positive.

The positive ELISA results were confirmed with Westernblot (DRG GmbH, Marburg, Germany) and/or EcoLine assay (Genzyme Virotech, Rüsselsheim, Germany) from the same sample, always interpreted according to the manufacturers criteria. The patients with cns neuroborreliosis had specific csf antibodies detected by ELISA, and intrathecal antibody synthesis confirmed with Eco-line immunoblot. The control group (C) consisted of 7 healthy persons, seronegative to $B$. burgdorferi, with no active infection nor inflammation.

The study was approved by Ethical Board of the Medical University in Białystok, and the patients gave informed written consent for participation.

On admission, $8 \mathrm{ml}$ of blood were drawn into heparincoated tubes and within 30 minutes centrifuged in Gradisol L (Aqua Medica, Poland) at 400g for $30 \mathrm{~min}$. PBMC fraction was pipetted, suspended in RPMI 1640 (Biomed, Poland), centrifuged and re-suspended in $1 \mathrm{ml}$ RPMI 1640 with 10\% inactivated bovine serum, streptomycin and penicillin. B. burgdorferi suspension of $10^{8}$ live bacteria/ml (Department of Rickettsiae, Chlamydiae and Zoonotic Spirochetes, National Institute of Hygiene, Warsaw), kept at $-70^{\circ} \mathrm{C}$ and thawed in room temperature directly before use. PBMC were cultured in sterile tubes in $5 \% \mathrm{CO}_{2}$ at $37^{\circ} \mathrm{C}$ in a concentration of $7.5 \times 10^{6}$ cells $/ \mathrm{ml}: 1$ ) in pure medium; 2) with spirochete strains B. garinii VS 46110, B. afzelii 20047 and B. burgdorferi sensu

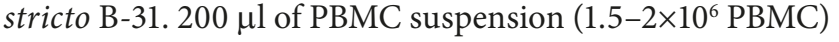
and $200 \mu \mathrm{l}$ of $B$. burgdorferi suspension $\left(2 \times 10^{7}\right.$ bacteria) was added to $600 \mu \mathrm{l}$ of medium to obtain multiplicity of infection (MOI) on the order of 10:1. Samples from all the subjects were incubated with $B$. burgdorferi ss, while, because of the limited cell yield, $B$. afzelii stimulation was performed in 3 patients with Lyme arthritis, 4 with neuroborreliosis, 3 with ACA and 4 controls, and B. garinii stimulation in 3 patients with Lyme arthritis, 3 with neuroborreliosis, 2 with ACA and 3 controls.

After 48-hours incubation, culture supernatant was pipetted, frozen at $-80^{\circ} \mathrm{C}$ and stored until thawed for further study. Concentrations were measured simultaneously in all the samples with commercial enzyme-linked immunosorbent assays (ELISA), IL-10 with an assay from BD Biosciences (San Jose, USA), IL-17A, IL-17F and IL-22 with assays from DRG GmbH (Marburg, Germany). The laboratory procedures and calculation of the results were performed according to the manufacturers' instructions. The detection limit was $2 \mathrm{pg} / \mathrm{ml}$ for IL-10, $0.5 \mathrm{pg} / \mathrm{ml}$ for IL-17A, $15.5 \mathrm{pg} / \mathrm{ml}$ for IL$17 \mathrm{~F}$ and $5.0 \mathrm{pg} / \mathrm{ml}$ for IL-22, and the read-outs below that were considered 0 .

Analysis was performed with Statistica 9.0. software. Comparisons between the Lyme borreliosis and control groups were performed with U Mann-Whitney test, and between the groups of patients with different forms of Lyme borreliosis with Kruskal-Wallis ANOVA. Comparisons between cultures (non-stimulated and stimulated with different species) were performed with Friedmann ANOVA, in Lyme borreliosis and control groups separately. Correlations were assessed with $\mathrm{R}$ Spearman test. $\mathrm{P}<0.05$ was considered significant. 


\section{RESULTS}

Supernatant concentrations of IL-17A, -17F and -22 are shown in Figures 1, 2 and 3, respectively. Median concentrations were increased significantly in stimulated, compared with unstimulated cultures in the Lyme borreliosis group. The same trend was present in healthy controls, not achieving a statistical significance, possibly because of the smaller size of the group. There was no difference between Lyme borreliosis and control groups, neither with nor without stimulation. The median IL-17F concentration was a few times higher than that of IL-17A.

In the group with Lyme borreliosis, the concentration of IL-17A was significantly higher under stimulation with B. afzelii than with B. burgdorferi ss (Fig. 1). IL-17F and IL-22 expression did not depend significantly on the stimulating species (Figs. 2, 3).

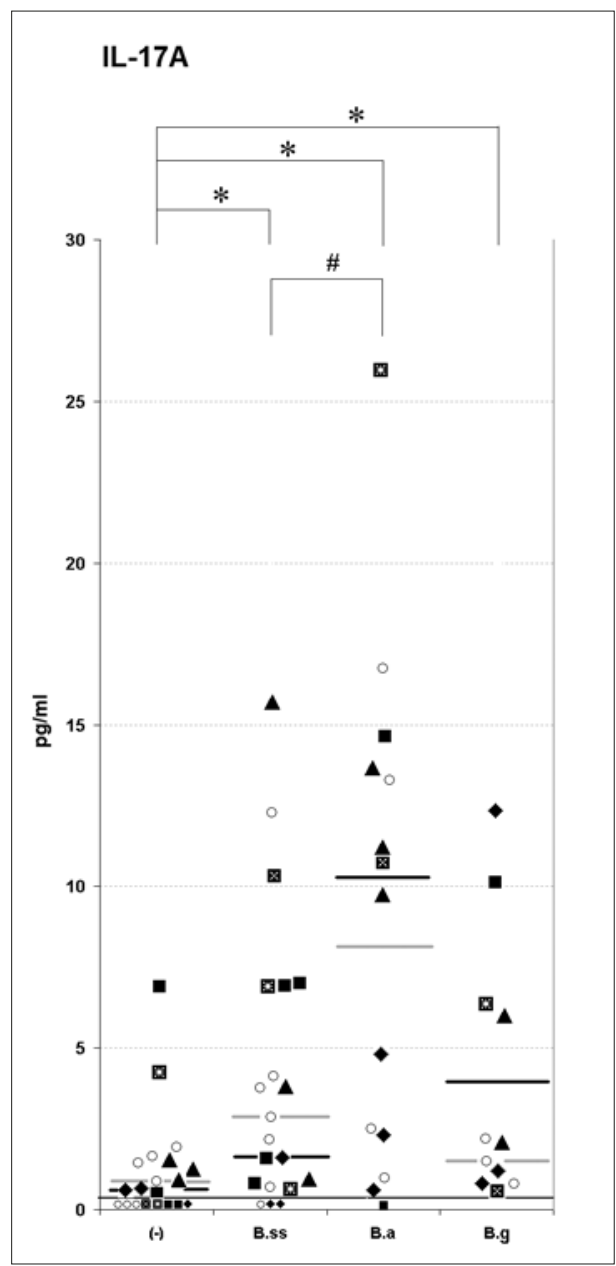

Figure 1. Concentration of IL-17A in the supernatant of PBMC cultures incubated for 48 hours, expressed in $\mathrm{pg} / \mathrm{ml}$.

Values from individual cultures: (-) - non-stimulated cultures: B.ss. - stimulation with B. burgdorferi ss, B.a. - stimulation with B. afzelii, B. g. - stimulation with B. garinii; $\bigcirc$ - healthy controls; - patients with Lyme arthritis; - patients with neuroborreliosis ( peripheral neuroborreliosis without cns involvement, $\mathbf{X}$-late cns neuroborreliosis, filled squares - early cns neuroborrelisis); $\boldsymbol{\Delta}$ - patients with ACA.

Horizontal line at the bottom denotes detection level. Thick horizontal lines represent median in Lyme borreliosis (black) and control (grey) groups. For Lyme borreliosis group: * - significant $(P<0.05)$ difference in comparison with $(-)$ $\#$ - significant $(P<0.05)$ difference between B.a. and B.ss.

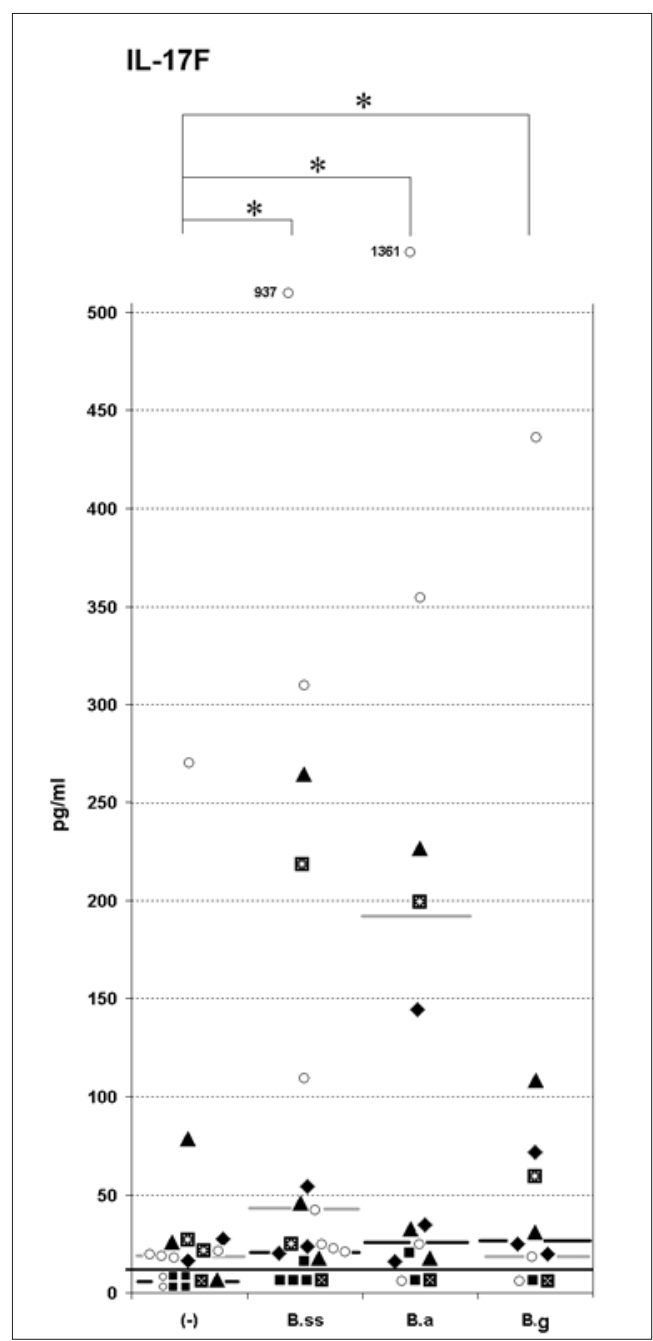

Figure 2. Concentration of IL-17F in the supernatant of PBMC cultures incubated for 48-hours, expressed in $\mathrm{pg} / \mathrm{ml}$.

Values from individual cultures: (-) - non-stimulated; B.ss. stimulation with B. burgdorferi ss, B.a. - stimulation with B. afzelii, B. g. - stimulation with B. garinii; $O$ - healthy controls; patients with Lyme arthritis; - patients with neuroborreliosis (* - peripheral neuroborreliosis without cns involvement, $\mathbf{X}$ - late cns neuroborreliosis, filled squares - early cns neuroborrelisis); $\Delta$ - patients with ACA.

Horizontal line at the bottom denotes detection level. Thick horizontal lines represent median in Lyme borreliosis patients (black) and healthy controls (grey). Individual extremely high values are shown out of scale above the plot. For Lyme borreliosis group: * significant $(P<0.05)$ difference in comparison with $(-)$

Because there was no difference between the levels of IL-17F and IL-22 induced by different $B$. burgdorferi species, in the following analysis of the expression of these 2 cytokines, the results from all the stimulated cultures were pooled, irrespective of the species. This increased the sample number allowing for the comparison of IL-17F and IL-22 concentrations between patients with different clinical forms of Lyme borreliosis. There was a lower concentration of IL-17F in neuroborreliosis patients than in controls, and than in patients with other forms of Lyme borreliosis (Fig. 4). The difference depended on the data from 4 patients with early cns neuroborreliosis, in whom IL-17F concentration was either undetectable or very low. No similar difference was observed for IL-22.

IL-10 was detectable in all the non-stimulated cultures (median concentration $0.22 \mathrm{ng} / \mathrm{ml}$ in Lyme borreliosis and 


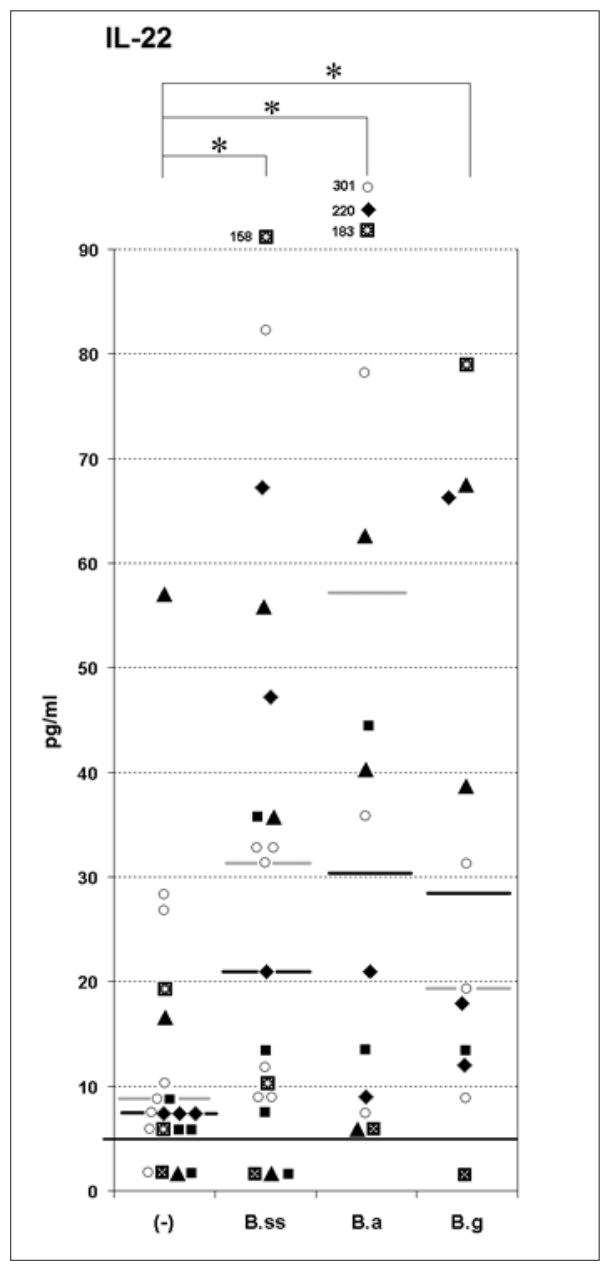

Figure 3. Concentration of IL-22 in the supernatant of PBMC cultures incubated for 48-hours, expressed in $\mathrm{pg} / \mathrm{ml}$. Values from individual cultures: (-) - non-stimulated; B.ss. - stimulation with B. burgdorferi ss, B.a. - stimulation with B. afzelii, B. g. - stimulation with B. garinii; $\bigcirc$ - healthy controls; $\bullet$ - patients with Lyme arthritis; - patients with neuroborreliosis ( - peripheral neuroborreliosis without cns involvement, $\mathbf{X}$ - late cns neuroborreliosis, filled squares - early cns neuroborrelisis); $\Delta$ - patients with ACA.

Horizontal line at the bottom denotes detection level. Thick horizontal lines represent median in Lyme borreliosis patients (black) and healthy controls (grey). Individual extremely high values were shown out of scale above the plot. For Lyme borreliosis group: * - significant $(P<0.05)$ difference in comparison with (-)

$0.51 \mathrm{ng} / \mathrm{ml}$ in controls) and up-regulated by stimulation to a median of $7.01 \mathrm{ng} / \mathrm{ml}, 6.22 \mathrm{ng} / \mathrm{ml}$ and $6.34 \mathrm{ng} / \mathrm{ml}$ in the Lyme borreliosis group in the presence of $B$. afzelii, B. garinii and $B$. burgdorferi ss, respectively. There was no correlation between the concentration of IL-10 and any of the Th17 cytokines.

\section{DISCUSSION}

Th17 lymphocytes, essential in the control of the bacterial infections, are also involved in the autoimmune inflammation in synovia and cns tissue $[7,8,10,12,16]$. In sclerosis multiplex, the fraction of IL-17A-producing csf lymphocytes is increased and IL-17A mRNA expression correlates with the clinical activity [16]. In rheumatoid arthritis (RA), IL-17Apositive cells are present in synovium infiltrate, co-localizing

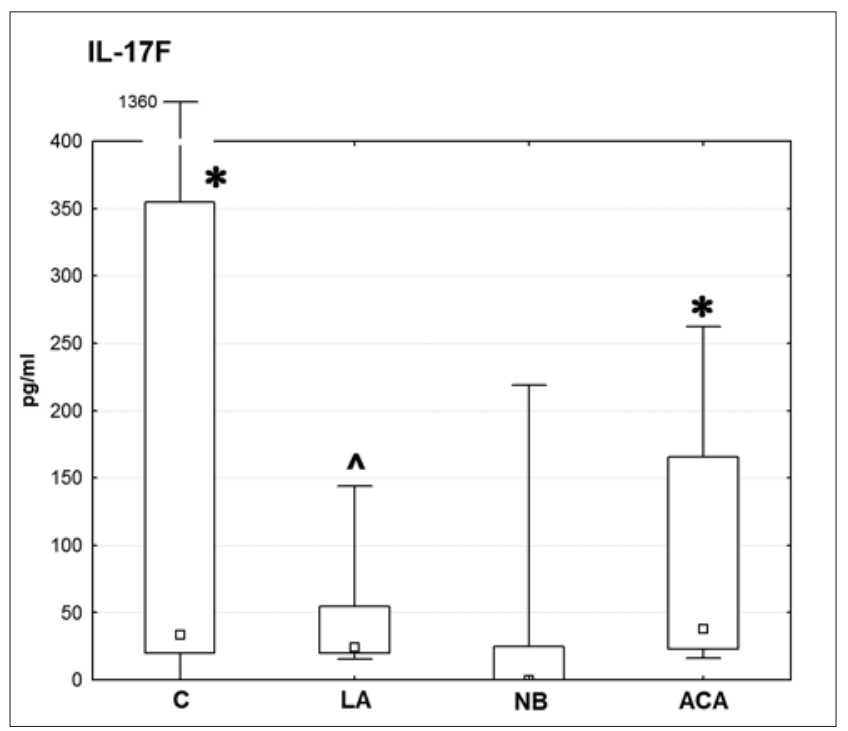

Figure 4. Concentration of IL-17F $(\mathrm{pg} / \mathrm{ml})$ in the supernatant of PBMC cultures incubated for 48-hours with B. burgdorferi spirochetes (combined data from all cultures stimulated with all three $B$. burgdorferi species, median shown as a square, quartiles as box, extreme values as whiskers, lower detection limit as horizontal line).

- cultures of PBMC from healthy controls $(n=14), L A$ - from patients with Lyme arthritis $(n=9), N B$ - from patients with neuroborreliosis $(n=14), A C A$ - from patients with ACA $(n=8)$; * - significantly higher than NB $(P<0.05) ; \wedge-$ higher than NB with $\mathrm{P}=0.06$

and cooperating with neutrophils [10]. IL-17F is expressed in RA alongside IL-17A, in a higher concentration that may compensate for its intrinsically weaker activity, and the receptor units for IL-17 on synoviocytes and infiltrating mononuclear cells are up-regulated and functional [12]. Intravenous administration of an anti-IL-17 antibody attenuates symptoms of RA [17]. Up-regulated IL-17A in synovial fluid and/or Th17 cells in the joints have been also detected in patients with reactive, psoriatic and juvenile idiopathic arthritis (JIA) [8, 18]. In JIA, the balance between immunomodulatory $\mathrm{T}_{\text {reg }}$ and pro-inflammatory Th17 lymphocyte populations determines the mild or severe clinical course [8]. IL-22 seems to contribute to Th17-driven autoimmunity, at least in some instances; for example, in lymphocytes from synovial fluid of JIA patients it is coexpressed with IL-17 [8].

Th17 cells participate in the defence against extracellular bacteria in skin and mucosa, which makes them likely to be engaged in the response to B. burgdorferi, an extracellular pathogen with a primary infection focus in the skin, at the tick bite site $[7,9]$. The IL-17A-producing lymphocyte population differentiates in vitro from naïve $\mathrm{T}$ cells under stimulation with sonicates and isolated lipopeptides of B. burgdorferi ss [6]. In the mouse model of Lyme arthitis, endogenous IL$17 \mathrm{~A}$ is necessary for the development of arthritis, while Th1 cytokines (INF- $\gamma$, IL-12) are not $[11,19]$.

Finally, IL-17A producing cells have been detected in the synovial fluid of the patients with Lyme arthitis [6]. IL$17 \mathrm{~A}$, most likely of intrathecal origin, is up-regulated in the csf of patients with early neuroborreliosis, correlating with pleocytosis $[14,15]$. Strle et al. detected IL-23, a cytokine crucial for the development of Th17 response, in serum of patients with EM, where it correlates with the presence of circulating autoantibodies and with the increased risk of residual symptoms lingering after the eradication of the infection [4]. 
In agreement with these results, in the presented study increased concentration of IL-17A was detected in the supernatant of PBMC cultures stimulated with B. burgdorferi. Moreover, extending previous observations on IL-17F, the role of which in Lyme borreliosis has not been studied so far, IL-17F was present in B. burgdorferi-stimulated cultures alongside IL-17A and in higher concentrations, resembling cytokine profile present in RA and, by analogy, suggesting that the role of IL-17A and IL-17F in Lyme borreliosis may be comparable and that they may act synergistically [12].

The current results confirm the presence of Th17 lymphocytes in the PBMC culture and their activation under stimulation. As Th17 memory cells are a part of the normal PBMC population, a small fraction of them should be present already at the start of the culture [6]. In Lyme borreliosis patients, this population could have already contained cells specifically recognizing $B$. burgdorferi antigens. However, the similarity of the response in Lyme borreliosis patients and seronegative controls suggests that it did not require any pre-existing immunity against $B$. burgdorferi and depended rather on unspecific, innate recognition of the pathogen. Actually, Th17 lymphocytes could have differentiated in the culture from the naïve T lymphocytes within the 48-hours long period of incubation, as a result of $B$. burgdorferi-elicited innate response [6].

The increased concentration of IL-10 is in an agreement with previous studies, confirming that this immunoregulatory/ Th2-related cytokine is expressed early in the innate response to $B$. burgdorferi, simultaneously with pro-inflammatory factors [20]. Unlike Th2 cytokines IL-4 and IL-13, IL-10 does not suppress Th17 lymphocyte differentiation, and its expression may coexist with a Th17-type response [10]; however, it might be able to modulate it differently in different culture conditions. In most studies on B. burgdorferi-elicited IL-17A and/or IL-10 expression, including the current study, the concentration of spirochetes was high, with MOI 10:1 or larger $[6,20]$. Bachmann et al. studied PBMC culture exposed to 2 orders of magnitude lower MOI (0.1:1), observing a qualitatively different cytokine expression pattern, with no IL-10 and IL-17A but with a vigorous IL-22 synthesis [13]. The later was inhibited by the addition of exogenous IL-10 and the authors suggest that IL-10 may block IL-22 expression when synthesized endogenously under stimulation with high spirochete MOI [13]. These data show that IL-22 could be up-regulated more effectively by low concentrations of spirochetes, which probably better reflect the situation in the infection focus in vivo. The lack of IL-10 synthesis in vivo could result in a paradoxically poorly controlled and persistent inflammation in response to low spirochete numbers. The presented results, however, do not confirm this assumption, as the synthesis of IL-22 continued at MOI 10:1, simultaneously with IL-17 and IL-10. There was no negative correlation between IL-10 and any of the Th17 cytokines, including IL-22. This shows that IL-22 expression may occur at a range of spirochete concentrations and that endogenous IL-10 is not always prohibitive to its synthesis. This, however, does not rule out Bachmann's et al. general conclusions about the different character of the Th17 response to different spirochete MOIs and its potential meaning for the pathogenesis of $B$. burgdorferi induced inflammation in vivo.

The concentration of IL-17F tended to be lower in the cultures from early neuroborreliosis patients. The difference was only significant after pooling samples stimulated with different $B$. burgdorferi species into a single group and needs confirmation in a larger study. However, it is highly consistent with the concept of Th17 response being related to a persistent inflammation in late Lyme borreliosis. Early neuroborreliosis is a self-limited disease [21], in which a vivid response against B. burgdorferi, including intrathecal expression of IL-17A, is followed by an effective immunomodulatory and antiinflammatory reaction $[14,15]$. The current data suggest that a relatively weak or strictly controlled systemic Th17 response may be a part of the inflammatory/immune background of this favourable course of the disease.

The inflammatory/immune response to B. burgdorferi ss is qualitatively similar, but stronger in comparison with B. afzelii and B. garini, which is reflected by a more active clinical course of $B$. burgdorferi ss infection and the risk of autoimmune complications [5,22]. This pattern was not observed in the expression of Th17-related cytokines in the presented study, as the level of IL-22 and IL-17F did not differ between the species and the concentration of IL-17A elicited by $B$. afzelii was even higher in comparison with $B$. burgdorferi ss. This could not be explained by the more likely exposition of patients to B. afzelii than to B.burgdorferi ss and the preferential immune response to this particular species, because a trend was also present in the healthy controls who were seronegative and had no history of $B$. burgdorferi infection. B. afzelii causes a relatively long-lasting and lowsymptomatic infection, with a classical manifestation being ACA, in which live spirochetes persist locally in the skin for years in spite of a systemic immune response [22]. It is unclear if and how higher IL-17A expression may be related to that characteristic. Further studying of such differences, however, may help further elucidate the clinical and pathophysiologic variability of Lyme borreliosis.

\section{CONCLUSIONS}

1. Th17/Th22 response seems to contribute to the persistent inflammation in Lyme borreliosis, with a significant role of IL-17F and IL-22 in addition to IL-17A.

2. There is no evident antagonism between the Th17 cytokines (including IL-22) and the simultaneous IL-10 expression in response to $B$. burgdorferi.

3. IL-17A expression is higher in the presence of $B$. afzelii than of B. burgdorferi ss.

4. These conclusions should be confirmed by further studies including larger groups of patients presenting with different clinical forms of Lyme borreliosis.

\section{REFERENCES}

1. Steere AC, Klitz W, Drouin EE, Falk BA, Kwok WW, Nepom GT, et al. Antibiotic-refractory Lyme arthritis is associated with HLA-DR molecules that bind a Borrelia burgdorferi peptide. J Exp Med. 2006; 203(4): 961-971.

2. Shin JJ, Glickstein LJ, Steere AC. High levels of inflammatory chemokines and cytokines in joint fluid and synovial tissue throughout the course of antibiotic-refractory Lyme arthritis. Arthritis Rheum. 2007; 56(4): 1325-1335.

3. Shen S, Shin JJ, Strle K, McHugh G, Li X, Glickstein LJ et al. T regulatory cell number and function in patients with antibiotic-refractory and antibiotic-responsive Lyme arthritis. Arthritis Rheum. 2010; 62(7): 2127-2137. 
4. Strle K, Stupica D, Drouin EE, Steere AC, Strle F. Elevated levels of IL23 in a subsets of patients with post-Lyme disease symptoms following erythema migrans. Clin Infect Dis. 2014; 58(3): 372-380.

5. Strle K, Drouin EE, Shen S, Khoury JE, McHugh G, Ružić-Sabljić E et al. Borrelia burgdorferi stimulates macrophages to secrete higher levels of cytokines and chemokines than Borrelia afzelii or Borrelia garinii. J Infect Dis. 2009; 200(12): 1936-1943.

6. Infante-Duarte C, Horton HF, Byrne MC, Kamradt T. Microbial lipopeptides induce the production of IL-17 in Th cells. J Immunol. $2000 ; 165(11)$ : 6107-6115.

7. Gaffen SL. An overview of IL-17 function and signaling. Cytokine 2008; 43(3): 402-407.

8. Nistala K, Moncrieffe H, Newton KR, Varsani H, Hunter P, Wedderburn LR. Interleukin-17-producing $\mathrm{T}$ cells are entiched in the joints of children with arthritis, but have a reciprocal relationship to regulatory T cell numbers. Arthritis Rheum. 2008; 58(3): 875-887.

9. Eyerich S, Eyerich K, Cavani A, Schmidt-Weber C. IL-17 and IL-22: siblings, not twins. Trends Immunol. 2010; 31(9): 354-361.

10. Chabaud M, Durand JM, Buchs N, Fossiez F, Page G, Frappart L et al. Human interleukin-17. A T cell-derived proinflammatory cytokine produced by the rheumatoid synovium. Arthritis Rheum. 1999; 42(5): 963-970.

11. Nardelli DT, Kevin Luk KH, Kotlosky NJ, Warmer TF, Torrealba JR et al. Role of IL-17, transforming growth factor-beta, and IL-6 in the development of arthritis and production of anti-outer surface protein $\mathrm{A}$ borreliacidal antibodies in Borrelia-vaccinated and -challenged mice. FEMS Immunol Med Microbiol. 2008; 53(2): 265-274.

12. Zrioual S, Ecochard R, Tournadre A, Lenief V, Cazalis MA, Miossec P. Genome-wide comparison between IL-17A- and IL-17F-induced effects in human rheumatoid arthritis synoviocytes. J Immunol. 2009; 182(5): 3112-3120.

13. Bachmann M, Horn K, Rudloff I, Goren I, Holdener M, Christen U et al. Early production of IL-22 but not IL-17 by peripheral blood mononuclear cells exposed to live Borrelia burgdorferi: the role of monocytes and interleukin-1. PLoS Pathogens 2010; 6(10): e1001144.
14. Henningsson AJ, Tjernberg I, Malmvall B-E, Forsberg P, Ernerudh J. Indications of Th1 and Th17 responses in cerebrospinal fluid from patients with Lyme neuroborreliosis: a large retrospective study. J Neuroinflam. 2011; 8: 36-46.

15. Nordberg M, Forsberg P, Johansson A, Nyman D, Jansson Ch, Ernerudh $\mathrm{J}$ et al. Cytotoxic mechanisms may play role in the local immune response in the central nervous system in neuroborreliosis. J Neuroimmunol. 2011; 232(1-2): 186-193

16. Matusevicius D, Kivisäkk P, He B, Kostulas N, Özenci V, Fredrikson S et al. Interleukin-17 mRNA expression in blood and CSF mononuclear cells is augmented in multiple sclerosis. Multiple Sclerosis 1999; 5(2): 101-104.

17. Genovese MC, van den Bosch F, Robertson A, Bojin S, Biagini IM, Ryan P et al. LTY2439821, a humanized anti-interleukin-17 monoclonal antibody, in the treatment of patients with rheumatoid arthritis. A phase I randomized, double blind, placebo-controlled, proof-of-concept study. Arthritis Rheum. 2010(4); 62; 929-39.

18. Lubberts E. Th17 cytokines and arthritis. Semin Immunopathol. 2010; 32(1): 43-53.

19. Kotloski NJ, Nardelli DT, Peterson SH, Torrealba JR, Warner TF, Callister SM et al. Interleukin-23 is required for development of arthritis in mice vaccinated and challenged with Borrelia species. Clin Vaccine Immunol. 2008; 15(8): 1199-1207.

20. Janský L, Reymanová P, Kopecký J. Dynamics of cytokine production in human peripheral blood mononuclear cells stimulated by LPS or infected by Borrelia. Physiol Res. 2003; 52(6): 593-598.

21. Henningsson AJ, Malmvall B-E, Ernerudh J, Matussek A, Forsberg P. Neuroborreliosis - an epidemiological, clinical and healthcare cost study from an endemic area in the south-east of Sweden. Clin Microbiol Infect. $2010 ; 16(8):$ 1245-1251.

22. Wilske B, Fingerle V, Schulte-Spechtel U. Microbiological and serological diagnosis of Lyme borreliosis. FEMS Immunol Med Microbiol. 2007; 49(1): 13-21. 